\title{
Benchmarks for antiretroviral therapy
}

\author{
Oren J. Cohen and Anthony S. Fauci
}

Commentary
See related article,
pages $777-782$.

Address correspondence to: Oren J. Cohen, National Institute of Allergy and Infectious Diseases, 31 Center Drive MSC 2520, Building 31, Room 7A05, Bethesda, Maryland 20892-2520, USA. Phone: (301) 435-8583; Fax: (301) 594-7047; E-mail: ocohen@nih.gov.

Sharp declines in HIV-related morbidity and mortality over the last 4 years are due in part to a fuller understanding of the pathogenesis of HIV infection, the availability of sensitive assays that measure viral replication in vivo, and the use of combinations of antiretroviral drugs that profoundly suppress viral replication for prolonged periods (i.e., highly active antiretroviral therapy, or HAART) (1,2). These welcome developments, however, have not come without a host of new caveats and cautions. Currently available antiretroviral drugs, although potent, are unable to inhibit viral replication completely (3-6); in addition, HIV is able to persist in latent and anatomically privileged reservoirs (7). HAART regimens are further handicapped by the myriad toxicities and drug interactions associated with their use. These factors, combined with the requirement that patients take a large number of pills, often in excess of 10 daily, make adherence to the regimens exceptionally difficult.

Perhaps the greatest obstacle to the optimal, judicious use of HAART is the uncertainty that pervades the decisionmaking process. Recommendations regarding when to initiate antiretroviral therapy, what drugs to use, and what constitutes treatment failure are based on an uncomfortable mixture of real data and expert opinion $(8,9)$. Encouraged by early hopes of HIV eradication, many clinicians strive at all costs to achieve undetectable levels of plasma viremia in their patients. Unfortunately, it is becoming increasingly clear that this strategy leads to frequent switching of HAART regimens, which rapidly exhausts effective treatment options.

In this issue of the JCI, Rizzardi and colleagues provide clinicians with a valuable benchmark that will help to optimize the use of HAART (10). The investigators studied 118 treatmentnaive patients with $\mathrm{CD}^{+} \mathrm{T}$ cells counts greater than 250 cells/ $\mu \mathrm{L}$ and levels of plasma viremia greater than 5,000
copies/mL who achieved an undetectable level (i.e., < 50 copies $/ \mathrm{mL}$ ) of plasma viremia on HAART. A baseline lymph-node biopsy was performed in 53 of these patients; baseline virologic and immunologic parameters in peripheral blood and lymphoid tissue were analyzed for their predictive value with regard to response to treatment. By between 2 and 24 weeks after therapy began, plasma viremia was undetectable in all cases. Some of the variability in the duration of the treatment needed may be due to the fact that 8 different regimens were employed in 5 different studies. However, independent of possible drug-specific effects, the number of cells in lymphoid tissue that expressed HIV RNA at baseline correlated strongly with the baseline levels of plasma viremia, and both of these variables were highly predictive of the duration of treatment necessary to suppress viremia.

The correlation between the number of cells expressing HIV RNA in lymphoid tissue and the level of plasma viremia extends previous observations and highlights an important facet of the pathogenesis of HIV infection (11). Earlier work had clearly established the role of lymphoid tissue as a major site of HIV replication in vivo $(12,13)$. Initial studies with HAART demonstrated that steady-state levels of plasma viremia derive largely from newly infected target cells that are in a rapid state of turnover (14-16). This conclusion follows from the rapid exponential decay of plasma viremia following initiation of HAART and the fact that the antiretroviral drugs employed (i.e., reverse transcriptase and protease inhibitors) block infection of new cells but do not affect viral RNA expression in cells that are already infected. Taken together, these previous findings suggest that constant infection of new target cells in lymphoid tissue is a major contributor to the level of plasma viremia in a patient; the observations of Rizzardi et al. lend further support to this hypothesis.
It should not be surprising that the time necessary to achieve an undetectable level of plasma viremia depends on the baseline level of plasma viremia and the number of cells in lymphoid tissue expressing HIV RNA. Exponential decay of plasma viremia for several weeks after initiation of HAART is followed by a slower, but also fairly constant second phase of decay. The steep exponential decay reflects the rapid turnover of infected $\mathrm{CD}^{+} \mathrm{T}$ cells, whereas the second-phase decay reflects attrition of longer-lived infected cells, such as macrophages (16). The exponential nature of the decay predicts that the higher the baseline level of plasma viremia, the longer it will take to achieve an undetectable level. Thanks to the robust data set in the study by Rizzardi et al., this prediction is confirmed and should prove to be a very useful benchmark for following the response to ini-

\section{$\mathrm{R}$ \\ izzardi and colleagues provide clinicians with a valuable benchmark that will help to optimize the use of HAART.}

tial antiretroviral therapy. Using the plasma viremia decay curve generated by the 118 study subjects, one can readily predict the time necessary to achieve an undetectable level given any baseline level of viremia. Thus, for a baseline level of plasma viremia of 1,000 HIV RNA copies $/ \mathrm{mL}$, a level of less than 50 copies/mL should be achieved after approximately 15 days of therapy; for a baseline of 10,000 copies/mL, approximately 7 weeks of therapy would be necessary; for a baseline of 100,000 copies/mL, 11.5 weeks; and for a baseline of 1,000,000 copies/mL, 16 weeks. The baseline number of cells expressing HIV 
RNA in lymphoid tissue was also an excellent predictor of the time necessary to achieve undetectable levels of plasma viremia. Given the cohort's mean number of HIV-expressing cells per area of lymphoid tissue (72 cells per $100 \mathrm{~mm}^{2}$ ), decay curves revealed that 83 days of treatment would be necessary to abolish detectable viremia. This estimate is in excellent agreement with the predicted 73 days of treatment necessary to achieve viremia of less than 50 copies $/ \mathrm{mL}$ from the cohort's mean baseline level of viremia $(\sim 60,000$ copies $/ \mathrm{mL})$.

How should the findings of Rizzardi and colleagues alter the care of HIVinfected individuals? Current guidelines recommend that a change in antiretroviral therapy be considered if plasma viremia remains in the detectable range 4 to 6 months after initiating therapy (8). This recommendation is greatly strengthened by the data of Rizzardi et al; only $1(0.8 \%)$ subject in the study failed to reach this benchmark by that time. Each time they initiate therapy on a patient, clinicians might be encouraged to consult Figure 3 in the article by Rizzardi et al., which depicts the estimated time to suppress plasma viremia. Both patient and physician might then have a more accurate expectation regarding the length of time that will be needed to achieve an undetectable level of viremia, and plans for follow-up testing of viral load can be made accordingly. However, variable responses should still be expected; in this regard, individuals with 100,000 copies/mL at baseline required approximately 3-21 weeks of therapy to achieve an undetectable viremia. Another caveat concerns the interpretation of data in patients with high baseline viremia, because only 2 patients in the cohort had baseline levels of viremia greater than $1,000,000$ copies/mL.

Several variables have strong predictive power for virologic success or failure during antiretroviral therapy. Baseline levels of viremia and numbers of $\mathrm{CD} 4^{+}$ $\mathrm{T}$ cells are both important prognosticators (17-20); higher levels of viremia and lower numbers of $\mathrm{CD} 4^{+} \mathrm{T}$ cells predict a shorter duration of viral suppression. The rate of decay of viremia and the depth of the nadir achieved are also prognostically important with regard to viral suppression (20-24). Data from the DELTA trial further suggest that the nadir level of viremia achieved on therapy is predictive of survival (25). The present report allows for the prediction of the time necessary to achieve a level of viremia less than 50 copies $/ \mathrm{mL}$; importantly, this time interval recently was shown to be another prognostic variable related to viral suppression (20).

These findings naturally raise questions about the possibility of aggressive intervention aimed at altering the variables associated with virologic success during initiation of antiretroviral therapy. One potentially beneficial strategy would apply an extremely aggressive antiretroviral drug regimen early in treatment, in an attempt to maximize the rate of decay of viremia. A standard regimen could be substituted once viremia reached undetectable levels. Another intervention to consider could apply to individuals experiencing a suboptimal rate of decay of viremia after initiation of therapy. Drug-resistance testing could be useful in this setting to rule out the possibility that antiretroviral therapy had selected for a drugresistant virus. If there were no signs of emerging drug resistance, another antiretroviral drug might be added to the regimen in an effort to accelerate the decline in plasma viremia. These approaches should be tested in an effort to maximize the benefits of antiretroviral therapy, but the temptation to treat numbers rather than individuals must be avoided, and long-term clinical correlative data are imperative.

\section{Acknowledgments}

The authors are Executive Secretary and Co-Chair, respectively, of the Department of Health and Human Services/Henry J. Kaiser Family Foundation Panel on Clinical Practices for the Treatment of HIV Infection in Adults and Adolescents. The opinions expressed in this article are solely those of the authors and are not official policy positions of the United States Government or the Panel referred to above.

1. Palella, F.J., Jr., et al. 1998. Declining morbidity and mortality among patients with advanced human immunodeficiency virus infection. N. Engl. J. Med. 338:853-860.

2. Mocroft, A., et al. 1998. Changing patterns of mortality across Europe in patients infected with HIV-1. EuroSIDA Study Group. Lancet. 352:1725-1730.

3. Natarajan, V., et al. 1999. HIV-1 replication in patients with undetectable plasma virus receiving
HAART. Highly active antiretroviral therapy. Lancet. 353:119-120.

4. Zhang, L., et al. 1999. Quantifying residual HIV-1 replication in patients receiving combination antiretroviral therapy. N. Engl. J. Med. 340:1605-1613.

5. Furtado, M.R., et al. 1999. Persistence of HIV-1 transcription in peripheral-blood mononuclear cells in patients receiving potent antiretroviral therapy. $N$. Engl. J. Med. 340:1614-1622.

6. Dornadula, G., et al. 1999. Residual HIV-1 RNA in blood plasma of patients taking suppressive highly active antiretroviral therapy. JAMA. 282:1627-1632

7. Chun, T.W., and Fauci, A.S. 1999. Latent reservoirs of HIV: obstacles to the eradication of virus. Proc. Natl. Acad. Sci. USA. 96:10958-10961.

8. Department of Health and Human Services/Henry J. Kaiser Family Foundation Panel on Clinical Practices for Treatment of HIV Infection. 1998. Guidelines for the use of antiretroviral agents in HIVinfected adults and adolescents. Ann. Intern. Med. 128:1079-1100. Current guidelines available at www.hivatis.org.

9. Carpenter, C.C., et al. 2000. Antiretroviral therapy in adults: updated recommendations of the International AIDS Society-USA Panel. JAMA. 283:381-390.

10. Rizzardi, G.P., et al. 2000. Predicting the duration of antiviral treatment needed to suppress plasma HIV1 RNA. J. Clin. Invest. 105:777-782.

11. Haase, A.T., et al. 1996. Quantitative image analysis of HIV-1 infection in lymphoid tissue. Science. 274:985-989.

12. Pantaleo, G., et al. 1993. HIV infection is active and progressive in lymphoid tissue during the clinically latent stage of disease. Nature. 362:355-358.

13. Embretson, J., et al. 1993. Massive covert infection of helper T lymphocytes and macrophages by HIV during the incubation period of AIDS. Nature. 362:359-362.

14. Wei, X., et al. 1995. Viral dynamics in human immunodeficiency virus type 1 infection. Nature. 373:117-122.

15. Ho, D.D., et al. 1995. Rapid turnover of plasma virions and CD4 lymphocytes in HIV-1 infection. Nature. 373:123-126.

16. Perelson, A.S., et al. 1997. Decay characteristics of HIV-1 infected compartments during combination therapy. Nature. 387:188-191.

17. Staszewski, S., et al. 1999. Virological response to protease inhibitor therapy in an HIV clinic cohort. AIDS. 13:367-373.

18. Deeks, S.G., et al. 1999. HIV RNA and CD4 cell count response to protease inhibitor therapy in an urban AIDS clinic: response to both initial and salvage therapy. AIDS. 13:F35-F43.

19. Lucas, G.M., Chaisson, R.E., and Moore, R.D. 1999 Highly active antiretroviral therapy in a large urban clinic: risk factors for virologic failure and adverse drug reactions. Ann. Intern. Med. 131:81-87.

20. Powderly, W.G., et al. 1999. Predictors of optimal virological response to potent antiretroviral therapy. AIDS. 13:1873-1880.

21. O'Brien, W.A., et al. 1996. Changes in plasma HIV-1 RNA and CD4+ lymphocyte counts and the risk of progression to AIDS. N. Engl. J. Med. 334:426-431.

22. Kempf, D.J., et al. 1998. The duration of viral suppression during protease inhibitor therapy for HIV1 infection is predicted by plasma HIV-1 RNA at the nadir. AIDS. 12:F9-F14.

23. Raboud, J.M., et al. 1998. Suppression of plasma viral load below 20 copies $/ \mathrm{ml}$ is required to achieve a long-term response to therapy. AIDS. 12:1619-1624.

24. Pilcher, C.D., et al. 1999. Detectable HIV-1 RNA at levels below quantifiable limits by amplicor HIV-1 monitor is associated with virologic relapse on antiretroviral therapy. AIDS. 13:1337-1342.

25. Delta Coordinating Committee and Delta Virology Committee. 1999. HIV-1 RNA response to antiretroviral treatment in 1280 participants in the Delta Trial: an extended virology study. AIDS. 13:57-65. 